\title{
A model of a dc silane discharge
}

\author{
D. J. Koch a) and W. N. G. Hitchon \\ University of Wisconsin-Madison, Department of Electrical and Computer Engineering, and the \\ Engineering Research Center for Plasma-Aided Manufacturing, Madison, Wisconsin 53706
}

(Received 30 November 1990; accepted for publication 22 July 1991)

\begin{abstract}
This paper describes a novel approach to modeling chemically active dc discharges, and its application to silane. An appropriate electric field was determined from a combination of experimental results and classical dc discharge theory. Utilizing this electric field, a kinetic description of the electron motion was used to calculate the ionization rate and the electron distribution function throughout the discharge. From the calculated rate of ionization and the assumed electric field and allowing for chemical reactions, steady-state fluxes of ions to the anode were calculated. These results compared favorably to the experimental results of Weakleim et al. [J. Vac. Sci. Technol. A 1, 29 (1987)]. A proposed model for the observed variations in the fractional ion currents is given.
\end{abstract}

\section{INTRODUCTION}

In this paper, a technique for describing chemically active dc discharges is presented and applied to silane. Silane discharges are technologically relevant for the deposition of amorphous hydrogenated silicon $(a-\mathrm{Si}: \mathrm{H})$ used for photovoltaics and other microelectronic applications. In dc silane discharges, deposition is performed on the anode surface since sputtering due to ion bombardment is negligible there. As a first step to understanding the deposition process, one must know the fluxes and energies of various ions and neutrals that impinge on the deposition surface. This paper describes a model for the entire discharge but will focus upon calculating the composition of the different ionic species that reach the anode. Comparisons with the limited available experimental data are made.

A detailed and electrostatically self-consistent calculation would be required in order to simulate all processes to steady state. In the case of silane, the slowest relevant process is charged particle recombination which requires approximately $5 \mathrm{~ms}$ to reach steady state. ${ }^{1}$ Since the calculations require time steps on the order of the electron plasma period $\left(<10^{-9} \mathrm{~s}\right)$, such a simulation would require over $10^{6}$ iterations. This calculation is not only time consuming, but would be difficult to justify since the errors in the chemical rate constants are large enough to nullify any accuracy gained in doing an electrostatically self-consistent calculation.

To overcome this obstacle, and since there is also some indication that this problem requires at least two spatial dimensions for a complete description, approximations are appropriate. In this work, an approximate electric field derived from the literature ${ }^{2}$ and experiment $t^{3,4}$ is imposed, from which the discharge properties are calculated. From the calculated discharge parameters, improvements to the assumed electric field configuration can be made and the discharge parameters are again found.

The structure of this paper is as follows. In Sec. II, the method for obtaining the electric field is given. Results of

\footnotetext{
") Present address: IBM-Rochester, Hwy. 52 and N.W. 37th St., Rochester, MN 55901.
}

the calculation for a dc silane discharge are given in Sec. III. The effects of varying the electrode spacing and applied potential on the fluxes of ions at the anode and a comparison with the experimental data of Weakleim ${ }^{3}$ are made. Studies of the ion flux variations due to changing assumptions made about the electric field and chemical rate constants are also done. A proposed mechanism for the effects of the electron temperature on the fractional ion current is given, followed by a summary of the results obtained.

\section{COMPUTATIONAL METHOD}

\section{A. Electric field determination}

As stated, the electric field is determined from a combination of experiments, ${ }^{3,4} \mathrm{dc}$ discharge theory, ${ }^{2}$ and calculated electron properties. In this model, the discharge will be divided into three regions: the cathode fall (and negative glow), the positive column, and the anode sheath (see Fig. 1). In this section, the method for determining the electric field in each region is given.

\section{Cathode fall}

Since the current and pressure are constant throughout the experiments to be simulated, the cathode fall potential drop and length are assumed to be constant. ${ }^{2}$ Weakleim ${ }^{3}$ stated that when the electrode spacing was decreased below some minimum value, there was a sharp increase in the applied potential. This suggests that the cathode fall length is approximately this minimum electrode spacing and that below the minimum electrode spacing the cathode fall voltage must increase in order to have sufficient ionization to maintain the discharge. In the presented model, the cathode fall (which will include the negative glow) will be modeled by a linearly varying electric field with a potential $\operatorname{drop} \Phi_{\mathrm{cf}}=-575 \mathrm{~V}$ and a length $l_{\mathrm{cf}}=0.96 \mathrm{~cm}$.

\section{Positive column}

Electrons leaving the cathode fall consist of two groups. The first comprises electrons that have experienced many inelastic collisions and have a low energy. The sec- 


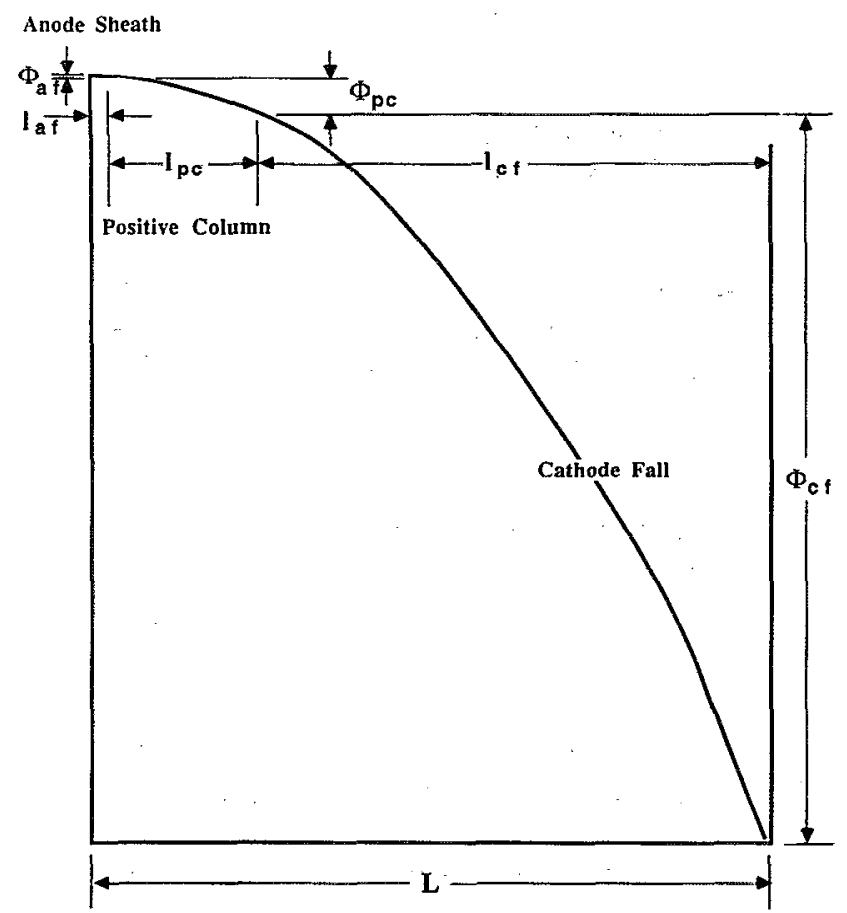

FIG. 1. Geometry of the dc discharge used here. $l_{a f}$ and $\Phi_{a f}$ are the length and potential drop of the anode sheath. $l_{\mathrm{pc}}$ and $\Phi_{\mathrm{pc}}$ are the length and potential drop of the positive column. $l_{\mathrm{cf}}$ and $\Phi_{\mathrm{cf}}$ are the cathode fall length and potential drop.

ond group consists of electrons that experience few inelastic collisions and enter the negative glow with energies directed along the discharge axis which are essentially as high as the cathode fall potential drop.

The calculations to be given show that in one particular case the electron energy is about $1 \mathrm{eV}$ with a beam component of energy along the discharge axis of $0.02 \mathrm{eV}$ at the position where the electric field approaches zero. This implies that the electron energy distribution is essentially isotropic in velocity space, which is very different from the "beamlike" distribution found in the cathode fall. Therefore, the region adjacent to the "cathode fall" will be treated in the electric field model as a resistive positive column. (As stated above, the term "cathode fall" will be used to describe both the cathode fall and negative glow.)

The experiments ${ }^{3}$ show that the applied voltage increases linearly with electrode spacing. It is assumed that this is in proportion with the length of the positive column which has a potential drop, $\Phi_{\mathrm{pc}}$, for a given electrode spacing, which is

$$
\Phi_{\mathrm{pc}}=\Phi_{c}-\Phi_{c \min }-\Phi_{a f}
$$

where $\Phi_{c}$ is the cathode potential at this electrode spacing, $\Phi_{a f}$ is the anode sheath potential, and $\Phi_{c \min }$ is the cathode potential at the minimum electrode spacing. The positive column extends from the negative glow edge to the edge of the anode sheath and its length is given by

$$
l_{\mathrm{pc}}=L-l_{\mathrm{cf}}-l_{a f}
$$

where $L$ is the electrode spacing, $l_{\mathrm{cf}}$ is the cathode fall length (including the negative glow), and $l_{a f}$ is the anode sheath length.

\section{Anode sheath}

Using simulations of the electron motion, the random electron current $J_{e r}$ and the drift current $J_{e d}$ can be calculated. In the cases studied here, the random current is much larger at the anode than the drift current. For current continuity to hold at the anode, an anode sheath is needed to reduce the incident discharge current to the external current. ${ }^{2}$ At the anode sheath-positive column boundary is a field reversal that marks the beginning of the anode sheath. Ions which are created in the anode sheath go to the anode while those ions created in the positive column are probably lost by Schottky diffusion processes to the chamber walls while ions created in the cathode fall go to the cathode.

The electron distribution in front of the anode is assumed here to consist of a Maxwellian component and a beam component. The temperature of the Maxwellian component $T_{e}$ is found from

$$
T_{e}=(2 / 3 k)\left(\left\langle E_{T}\right\rangle-\left\langle E_{B}\right\rangle\right),
$$

where $\left\langle E_{T}\right\rangle$ is the total average electron energy and $\left\langle E_{B}\right\rangle$ is the average electron beam energy which is relatively small. From $T_{e}$, the average electron velocity towards the anode $v_{e x}$ is found using the relation

$$
v_{e x}=\sqrt{k T_{e} / 2 \pi m_{e}},
$$

assuming the low-energy beam does not reach the anode and the distribution is still Maxwellian with temperature $T_{e}$ at the anode.

Knowing $J_{\text {ext }}$ and $v_{e x}$, the electron density at the anode can be determined and is

$$
\left.N_{e}\right|_{\text {anode }}=J_{\text {ext }} / q v_{e x} \text {. }
$$

The anode sheath potential drop is assumed to be $3.8 k T_{e} / q$, so the electron density at the anode fall-positive column interface can be estimated to be

$$
\left.N_{e}\right|_{\text {afpc }}=\left.N_{e}\right|_{\text {anode }} \exp (3.8) \text {. }
$$

From $T_{e}$ and $\left.N_{e}\right|_{a f \mathrm{pc}}, \lambda_{D}$ is calculated in the usual manner. The anode sheath $l_{a f}$ is assumed to extend $5 \lambda_{D}$ from the anode.

Using these calculated valucs of $\Phi_{a f}$ and $l_{a f}$, new values of $\Phi_{\mathrm{pc}}$ and $l_{\mathrm{pc}}$ are found. The electron motion is simulated in this new electric field configuration. The electron temperature at the anode after the second simulation was found to be within $15 \%$ of the initial electron temperature. The effect of the variation in the electron temperature on the ion current will be studied in Sec. III.

\section{B. Particle motion calculation}

Using this electric field and the method for calculating electron motion described in other work, ${ }^{5,6}$ the spatial distribution of the ionization and dissociation rates are deter- 
TABLE I. The ion-neutral chemical reactions and their respective rate constants used for modeling the ion motion in the anode sheath. Only exothermic and thermineutral reactions are used.

\begin{tabular}{|c|c|}
\hline Process & $\begin{array}{l}\text { Rate constant } \\
\left(\mathrm{cm}^{3} \mathrm{~s}^{-1)}\right.\end{array}$ \\
\hline $\mathrm{SiH}_{3}^{+}+\mathrm{SiH}_{4} \rightarrow \mathrm{Si}_{2} \mathrm{II}_{3}^{+}+2 \mathrm{II}_{2}$ & $0.07 \times 10^{-10}$ \\
\hline $\mathrm{SiH}_{3}^{+}+\mathrm{SiH}_{4} \rightarrow \mathrm{Si}_{2} \mathrm{H}_{5}^{+}+\mathrm{H}_{2}$ & $0.25 \times 10^{-10}$ \\
\hline $\mathrm{SiH}_{3}^{+}+\mathrm{SiH}_{4} \rightarrow \mathrm{Si}_{2}^{-} \mathrm{H}_{7}^{+}+\mathrm{H}_{4}$ & $1.8 \times 10^{-26}\left(\mathrm{~cm}^{6} \mathrm{~s}^{-1}\right)$ \\
\hline $\mathrm{SiHH}_{2}^{+}+\mathrm{SiH}_{4} \rightarrow \mathrm{SiH}_{3}^{+}+\mathrm{H}_{3}$ & $1.07 \times 10^{-9}$ \\
\hline $\mathrm{SiH}_{2}^{+}+\mathrm{SiH}_{4} \rightarrow \mathrm{Si}_{2} \mathrm{H}_{2}^{+}+2 \mathrm{H}_{2}$ & $0.55 \times 10^{-10}$ \\
\hline $\mathrm{SiHH}_{2}^{+}+\mathrm{SiH}_{4} \rightarrow \mathrm{Si}_{2} \mathrm{H}_{4}^{+}+\mathrm{H}_{2}$ & $2.50 \times 10^{-10}$ \\
\hline $\mathrm{SiH}^{+}+\mathrm{SiH}_{4} \rightarrow \mathrm{Si}_{2} \mathrm{H}^{+}+2 \mathrm{H}_{2}$ & $0.70 \times 10^{-10}$ \\
\hline $\mathrm{SiH}^{+}+\mathrm{SiH}_{4} \rightarrow \mathrm{Si}_{2} \mathrm{H}_{3}^{+}+\mathrm{H}_{2}$ & $2.80 \times 10^{-10}$ \\
\hline $\mathrm{SiH}^{+}+\mathrm{SiH}_{4} \rightarrow \mathrm{Si}_{2} \mathrm{H}_{5}^{+}+\mathrm{SiH}_{4}$ & $1.30 \times 10^{-26}\left(\mathrm{~cm}^{6} \mathrm{~s}^{-1}\right)$ \\
\hline $\mathrm{Si}^{+}+\mathrm{SiH}_{4} \rightarrow \mathrm{Si}_{2} \mathrm{H}_{2}^{+}+\mathrm{H}_{2}$ & $4.80 \times 10^{-10}$ \\
\hline $\mathrm{Si}^{+}+2 \mathrm{SiH}_{4} \rightarrow \mathrm{Si}_{2} \mathrm{H}_{4}^{+}+\mathrm{SiH}_{4}$ & $1.40 \times 10^{-26}\left(\mathrm{~cm}^{6} \mathrm{~s}^{-1}\right)$ \\
\hline $\mathrm{Si}_{2} \mathrm{H}_{7}^{+}+\mathrm{SiH}_{4} \rightarrow \mathrm{Si}_{3} \mathrm{H}_{6}^{+}+2 \mathrm{H}_{2}+\mathrm{H}$ & $5.00 \times 10^{-11}$ \\
\hline $\mathrm{Si}_{2} \mathrm{H}_{6}^{+}+\mathrm{SiH}_{4} \rightarrow \mathrm{Si}_{3} \mathrm{H}_{6}^{+}+2 \mathrm{H}_{2}$ & $5.00 \times 10^{-11}$ \\
\hline $\mathrm{Si}_{2} \mathrm{H}_{5}^{+}+\mathrm{SiH}_{4} \rightarrow \mathrm{Si}_{3} \mathrm{H}_{6}^{+}+\mathrm{H}_{2}+\mathrm{H}$ & $5.00 \times 10^{-11}$ \\
\hline $\mathrm{Si}_{2} \mathrm{H}_{4}^{+}+\mathrm{SiH}_{4} \rightarrow \mathrm{Si}_{3} \mathrm{H}_{6}^{+}+\mathrm{H}_{2}$ & $5.20 \times 10^{-11}$ \\
\hline $\mathrm{Si}_{2} \mathrm{H}_{3}^{+}+\mathrm{SiH}_{4} \rightarrow \mathrm{Si}_{3} \mathrm{H}_{6}^{+}+\mathrm{H}^{-}$ & $5.00 \times 10^{-11}$ \\
\hline $\mathrm{Si}_{2} \mathrm{H}_{2}^{+}+\mathrm{SiH}_{4} \rightarrow \mathrm{Si}_{3} \mathrm{H}_{6}^{+}$ & $3.76 \times 10^{-11}$ \\
\hline $\mathrm{Si}_{2} \mathrm{H}^{+}+\mathrm{SiH}_{4} \rightarrow \mathrm{Si}_{3} \mathrm{H}_{6}^{+}$ & $1.08 \times 10^{-10}$ \\
\hline $\mathrm{Si}_{m} \mathrm{H}_{n}^{+}+\mathrm{SiH}_{4} \rightarrow \mathrm{Si}_{m+I} \mathrm{H}_{n+2}^{+}+\mathrm{H}_{2}$ & $1.00 \times 10^{-11}$ \\
\hline
\end{tabular}

mined. The electron-silane collisions cross sections used were compiled by Ohmuri et al.

From the ionization rate as well as the chemical rate constants compiled by Kushner, ${ }^{1}$ from various experiments, ${ }^{8-12}$ the steady-state flux of the different species to the anode are calculated. The ion-silane chemical reactions considered here either had a small activation energy or were exothermic since the ion energies in the anode sheath are too small to activate highly endothermic reactions. These ion fluxes are compared to the experimental work of Weakleim et $a l^{3}$ and calculations by Kushner. ${ }^{1}$ (See Table I.)

\section{III. dc SILANE DISCHARGE CALCULATION}

In this section, the results using the technique given in Sec. II for a dc silane discharge are presented. The experiment that is modeled here was performed by Weakleim et $a l .^{3}$ The purpose of the experiment was to determine the effect of the elcctrode spacing on the current of various silane ion species at the anode. The discharge current remained constant since the applied voltage was varied to maintain the current $\left(0.13 \mathrm{~mA} / \mathrm{cm}^{2}\right)$. The purpose of this calculation is to model the experiment and to determine the cause of the variation in the ion currents to the anode.

This section begins with a brief exposition of the results of the electron motion calculation. This will include a study of the effects on the electron motion due to assumptions made as to the electric field. This will be followed by the results of the ion motion calculation in Sec. III B. The effects of errors in the electron temperature and in data such as chemical rate constants on the fractional ion current are shown. A proposed explanation for the observed variation in the ion current will also be given. This will be followed by the results of the neutral chemistry calculation
Plasma Potential vs $X$

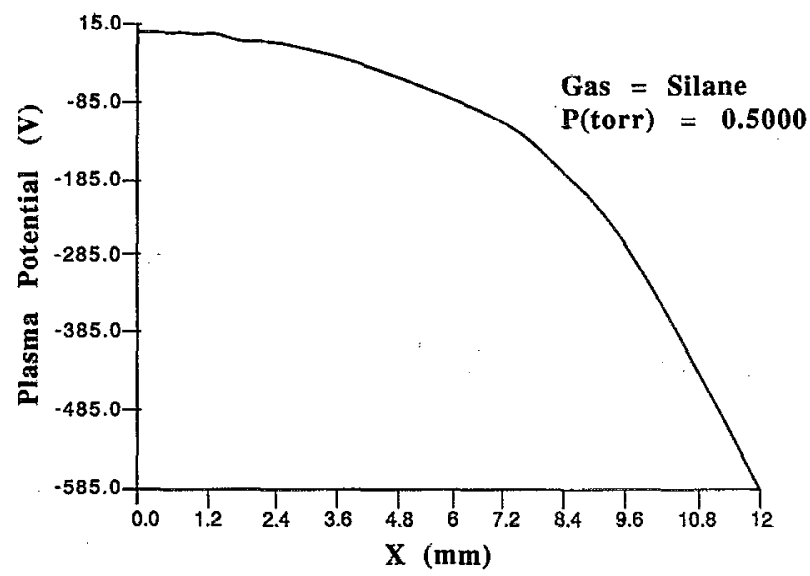

FIG. 2. Potential used for calculating the electron motion in a dc discharge with an electrode spacing, $L=1.2 \mathrm{~cm}$.

in Sec. III C. Since there are no experimental results on neutral behavior to compare with, a neutral motion calculation is done for only one electrode spacing.

\section{A. Electron motion results}

In this section, the results of the electron motion calculation are given. For each electrode spacing, the method described in Sec. II for modeling the electric field and electron motion was used to calculate the ionization rate and electron energy. The results of the electron motion calculation for an electrode spacing of $1.2 \mathrm{~cm}$ are given in Figs. 2-4. Figure 2 is a plot of the electrostatic potential used for an electrode spacing of $1.2 \mathrm{~cm}$. Figure 3 is the corresponding plot of the calculated average energy electron in the discharge. The ionization rate is shown in Fig. 4. This

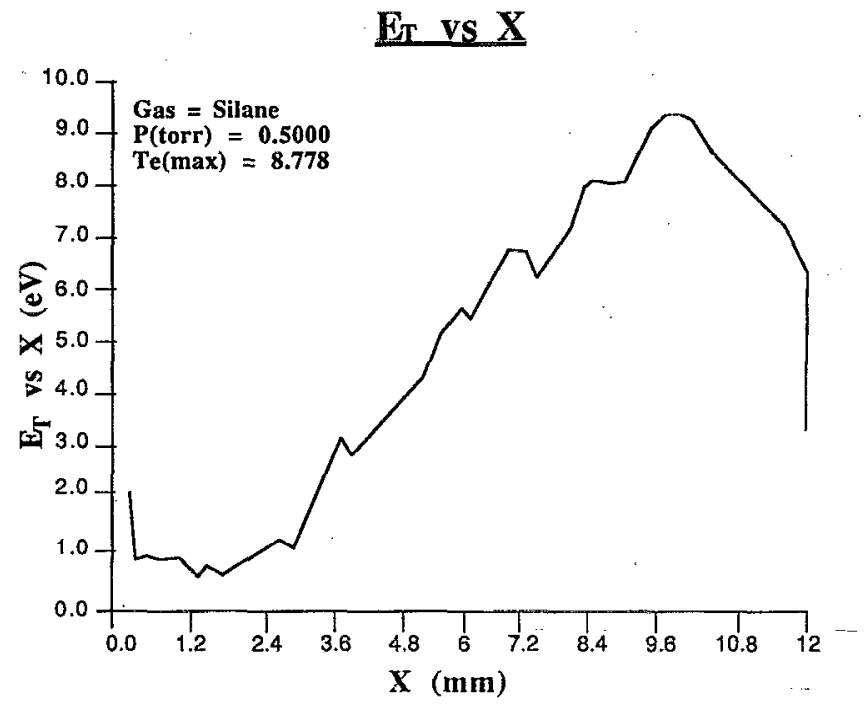

FIG. 3. Calculated average electron energy vs $x$ using the potential given in Fig. 2. 


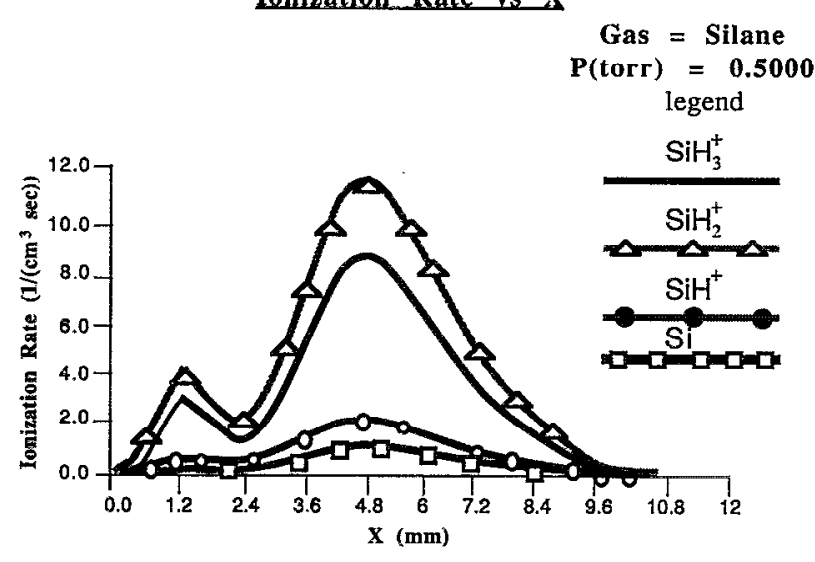

FIG. 4. Electron impact ionization rate of silane vs $x$ using the potential given in Fig. 2.

ionization rate is uscd as input for the ion motion calculation and the dissociation rate is used as input for the neutral chemistry calculation. Similar calculations were also done for electrode spacings of $L=1.0,1.1,1.4$, and $1.8 \mathrm{~cm}$.

As mentioned in Sec. II, the electric field model contains a field reversal at the positive column-anode sheath interface. The effect of this field is to trap electrons which have an energy less than $\left|q \Phi_{a f}\right|$ in the vicinity of the field inversion. In this region, the density of the confined electrons is much greater than the density of the electrons with an energy greater than $\left|q \Phi_{a f}\right|$. For a non-Maxwellian distribution, this can give a low $\left\langle E_{T}\right\rangle$ here.

The calculated electron energies at the anode, anode sheath potential and length for each electrode spacing are given in Table II. The electron energy at the anode increases with the length of the positive column, which increases with electrode spacing, due to the increased heating of the electrons by the positive column electric field. The heating (and cooling) of the high energy electron is reflected in the increase in the ionization and dissociation rates as these electrons travel through the positive column and the decreased rates in the anode sheath. The average electron energy at the anode begins to level off for electrode spacings of $1.4 \mathrm{~cm}$. The electron energy at the anode will play a vital role in determining the ion motion and the chemical reactions in the anode sheath as will be seen in the next section. Therefore, the accuracy of the method for determining the elcctron temperature must be examined.

TABLE II. Calculated values of the electron temperature at the anode, anode sheath length $l_{a f}$, and anode sheath potential drop $\Phi_{a f}$ for electrode spacings $L=1.0,1.1,1.2$, and $1.4 \mathrm{~cm}$.

\begin{tabular}{cccc}
\hline $\begin{array}{c}L \\
(\mathrm{~cm})\end{array}$ & $\begin{array}{c}T_{e} \\
(\mathrm{eV})\end{array}$ & $\begin{array}{c}\Phi_{a f} \\
(\mathrm{~V})\end{array}$ & $\begin{array}{c}l_{a f} \\
(\mathrm{~cm})\end{array}$ \\
\hline 1.0 & 0.133 & .505 & $1.76 \times 10^{-2}$ \\
1.1 & 0.272 & 1.03 & $3.00 \times 10^{-2}$ \\
1.2 & 1.460 & 5.75 & $1.03 \times 10^{-1}$ \\
1.4 & 1.550 & 5.80 & $1.10 \times 10^{-1}$ \\
\hline
\end{tabular}

TABLE III. The effects of changing the positive column potential drop on the electron temperature at the anode. ( $l_{p c}$ is held constant.)

\begin{tabular}{ccc}
\hline \hline $\begin{array}{c}\text { Applied voltage } \\
(\mathrm{V})\end{array}$ & $\begin{array}{c}\Phi_{p c} \\
(\mathrm{~V})\end{array}$ & $\begin{array}{c}T_{e} \\
(\mathrm{eV})\end{array}$ \\
\hline-585.0 & -15.5 & 1.46 \\
-581.5 & -12.0 & 1.32 \\
-579.0 & -9.50 & 1.26 \\
\hline
\end{tabular}

\section{Electron temperature varlations}

In this section, an estimate of the possible error in the calculated electron temperature is made. This error can have many origins. These include the assumed position of the negative glow-positive column boundary, the applied potential and the assumed anode sheath potential. First, we investigate the errors due to the assumed anode sheath potential, followed by the errors in the electron temperature due to changes in the length of the negative glow and the effect of crrors in the applied potential.

The first source of error in the electron temperature to be examined is the assumed anode sheath potential used in the electron simulation. The manner in which this error arises is that in the initial electron simulation, an assumed anode sheath potential and length are used. From this electric field configuration, the electron motion and subsequently the electron temperature at the anode are calculated. Using this temperature, a new anode sheath potential and length are found. The electron motion is again calculated using this electric field and the electron temperature is found.

The variation in the electron temperature between the two simulations is a measure of the uncertainty in the electron temperature discussed here. In these calculations, the maximum variation in the electron temperature due to varying the assumed sheath was found to be about $15 \%$ of the original values, which is quite small.

The second origin of possible error in the electron temperature calculation is the position of the negative glowpositive column boundary. The effect of this boundary is to change the electric field in the positive column (since $\Phi_{\mathrm{pc}}$ is constant). The changes in the electric field will alter the heating rate of the electrons which in turn changes the electron temperature at the anode sheath.

A simulation of the electron motion was done for an electrode spacing of $L=1.2 \mathrm{~cm}$ but where the positive column length was reduced by $40 \%$ from its original value. It was found that the electron temperature at the anode increased $15 \%$ from original value, which is again small.

The third source of error in the electron temperature is the measurement of the applied potential. Since the cathode fall potential is assumed constant (under the conditions of constant pressure and current) the effect of errors in the applied potential measurements is to change the electric field in the positive column. This effect is studied by changing $\Phi_{\mathrm{pc}}$ (and subsequently the applied voltage) using the original positive column length.

The example used here is for an electrode spacing of $L=1.2 \mathrm{~cm}$. The positive column potential was decreased 
Measured Fractional Ion Flux

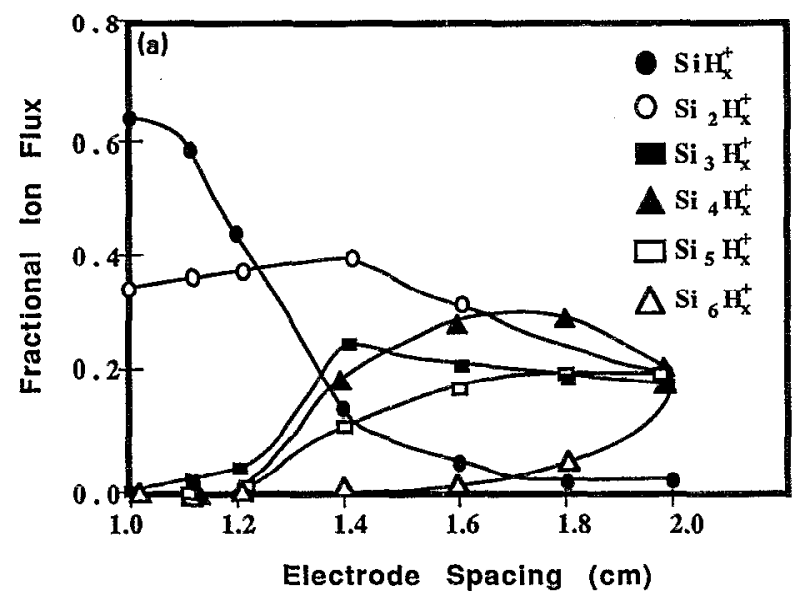

Calculated Fractional Ion Flux

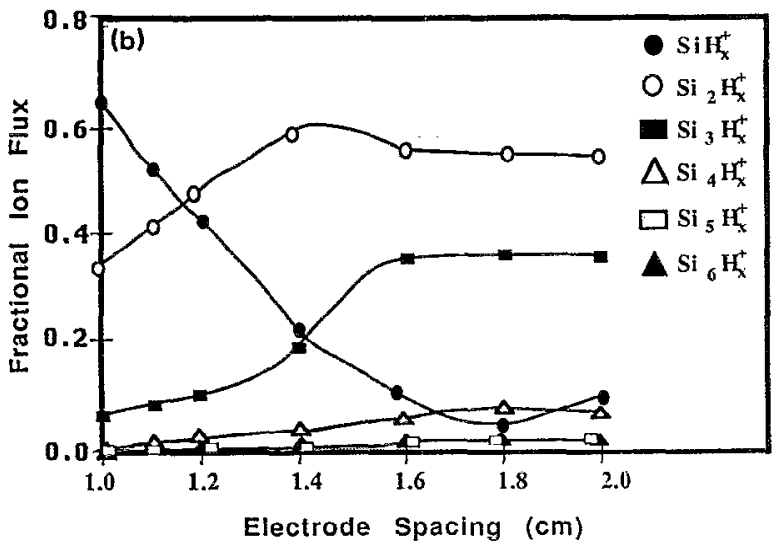

FIG. 5. Fractional ion currents to the anode vs electrode spacing $L$. (a) The measured values and (b) the values yielded by the code.

from -14.4 to $-9.5 \mathrm{~V}$ and electron simulations were performed. The results of these simulations are given in Table III. It was found that the electron temperature decreased by about $20 \%$ from the original value. This too is a relatively small effect. As we shall see in the next section, however, errors in the applied potential are more important for smaller electrode spacings.

\section{B. Ion motion results}

In this section, the results of the ion motion calculations are presented. These results will include an examination of the effects on the ion current due to changes in the electron temperature and ion-silane chemical rate constants.

Given the calculated ionization rate and anode sheath electric field and length, the fractional currents at the anode can be determined. The calculated and measured ${ }^{3}$ frac- $^{-}$ tional ion currents for each electrode spacing are given in Fig. 5. In the notation used here, $\mathrm{SiH}_{x}^{+}$denotes the sum of all ion currents in which there is one silicon atom in the ion. Likewise, $\mathrm{Si}_{2} \mathrm{H}_{x}^{+}$denotes the sum of all ion currents in which there are two silicon atoms in the ion.
$\mathrm{SiH}_{x}^{+}$Flux Comparison

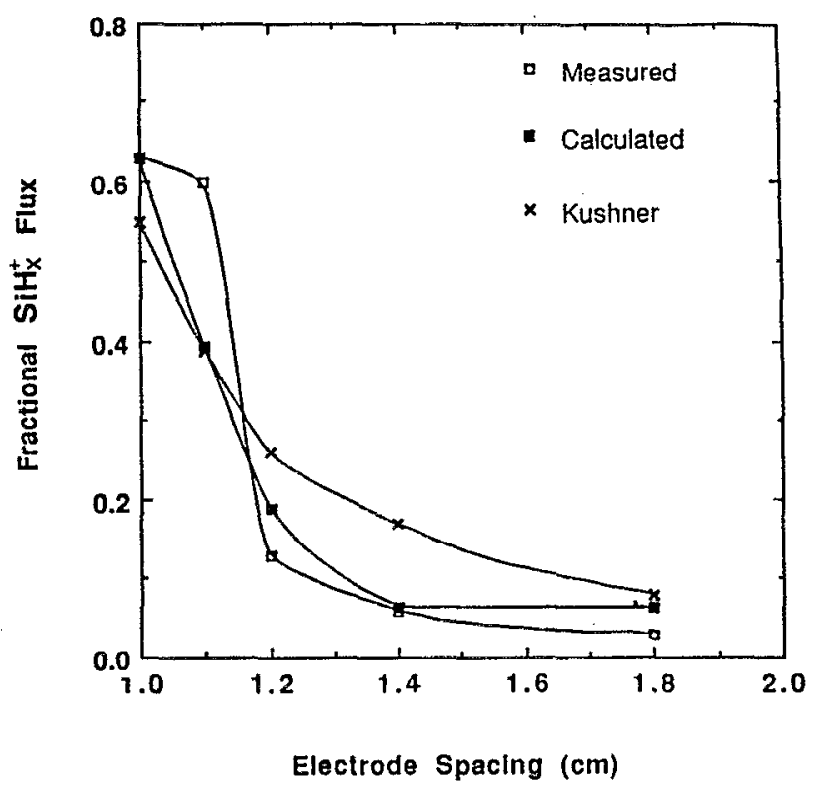

FIG. 6. A comparison of the fractional $\mathrm{SiH}_{x}^{+}$ion current vs electrode spacing: calculated, measured, and Kushner's results.

The $\mathrm{SiH}_{x}^{+}$current calculations have two main origins for error: the anode sheath electric field (principally via $T_{e}$ ) and the chemical rate constants used. The errors in the calculated currents of heavier ions such as $\mathrm{Si}_{2} \mathrm{H}_{x}^{+}$are due to the previously mentioned causes and to the errors in the chemical rate constants for creation and destruction of the higher silane ion species. These rate constants are estimates ${ }^{1}$ and probably have large errors.

Therefore, to compare the model with experiment, it is better to use the $\mathrm{SiH}_{x}^{+}$ion flux. Comparisons of the heavier silane ion currents will reveal information about the chemical rate constants for formation of those ions. Figure 6 gives a comparison of the fractional $\mathrm{SiH}_{x}^{+}$currents measured, calculated and given by Kushner. ${ }^{1}$ This theory and the experimental values are in good agreement for electrode spacings of $L=1.0,1.2,1.4$, and $1.8 \mathrm{~cm}$ and in moderate agreement at $L=1.1 \mathrm{~cm}$. The lack of agreement at $L=1.1 \mathrm{~cm}$ may be due to the possible errors in the positive column potential drop and will be discussed later. The discrepancy between the work by Kushner ${ }^{1}$ and experiment is probably due to the simple description of the electric field he used. We will now investigate the effects of errors in the calculated electron temperature and ion-silane chemical rate constants on the fractional $\mathrm{SiH}_{x}^{+}$flux.

\section{Ion flux variations due to electron temperature errors}

The first source of error in the fractional $\mathrm{SiH}_{x}^{+}$ion current considered here is the calculated electron temperature. The causes for the variations in the electron temperature were discussed in the previous section and will not be elaborated upon further. Here, the effects of changes in the electron temperature on the ion current are studied. 
$\mathrm{SiH}_{\mathrm{x}}^{+}$Flux vs. $\mathrm{L}$ and $\mathrm{T}$

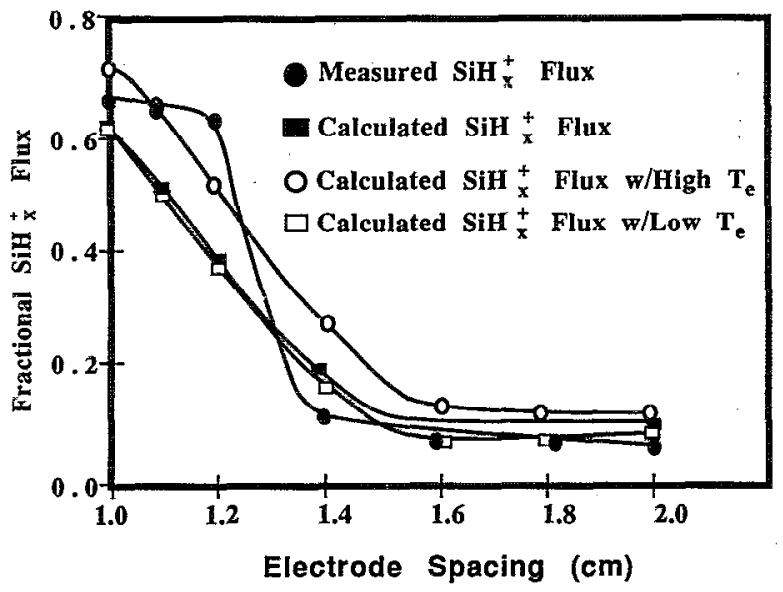

FIG. 7. Effects of the variations in the calculated $T_{e}$ on the fractional ion current at the anode. In this calculation, $T_{e}$ was allowed to vary $15 \%$ above and below the calculated $T_{e}$.

As mentioned in Sec. II A, the variation in $T_{e}$ due to the way in which the anode sheath potential is modeled can be as high as $15 \%$. Figure 7 shows the sensitivity of the $\mathrm{SiH}_{x}^{+}$current to the variations in electron temperature. The ion current was calculated using electron temperatures $15 \%$ above and below the calculated $T_{e}$ as well as the calculated and measured values. The fractional ion currents werề found to vary by less than $10 \%$ at all electrode spacings considered while the trends of the results were maintained.

Upon examining this plot, the discrepancy in the $\mathrm{SiH}_{x}^{+}$current at $L=1.1 \mathrm{~cm}$ cannot be explained by this mechanism alone. The only other way to recreate the measured fractional ion flux for an electrode spacing of $L=1.1$ $\mathrm{cm}$ is to reduce the electron temperature to approximately the value at $L=1.0 \mathrm{~cm}$. This can be accomplished by reducing the positive column potential drop. A reduction of the positive column potential drop from -5.5 to -2.5 $\mathrm{V}$ achieved the desired result, so it is plausible that an error in measuring the applied potential could have occurred. The electron temperature decreased from 0.272 to 0.177 $\mathrm{eV}$. The results of the ion flux simulation are given in Fig. 8 . This suggests that for short positive columns, measurement errors on the order of a few volts can drastically change the outcome of the simulation.

\section{Chemical rate constant uncertainties}

The second origin of error for the $\mathrm{SiH}_{x}^{+}$current is the chemical rate constants. From Fig. 4, the dominant products of the ionization of silane are $\mathrm{SiH}_{2}^{+}$and $\mathrm{SiH}_{3}^{+}$. From the table of ion chemical reactions (Table II), $\mathrm{SiH}_{2}^{+}$reacts quickly with silane to form $\mathrm{SiH}_{3}{ }^{+}$. The main mechanism for the loss of $\mathrm{SiH}_{3}^{+}$under these experimental conditions is

$$
\mathrm{SiH}_{3}^{+}+2 \mathrm{SiH}_{4} \rightarrow \mathrm{Si}_{2} \mathrm{H}_{7}^{+}+\mathrm{SiH}_{4} \text {. }
$$

The rate constant for this reaction was varied by $33 \%$ from the value quoted in the literature and the ion currents
Fractional Ion Flux Comparison

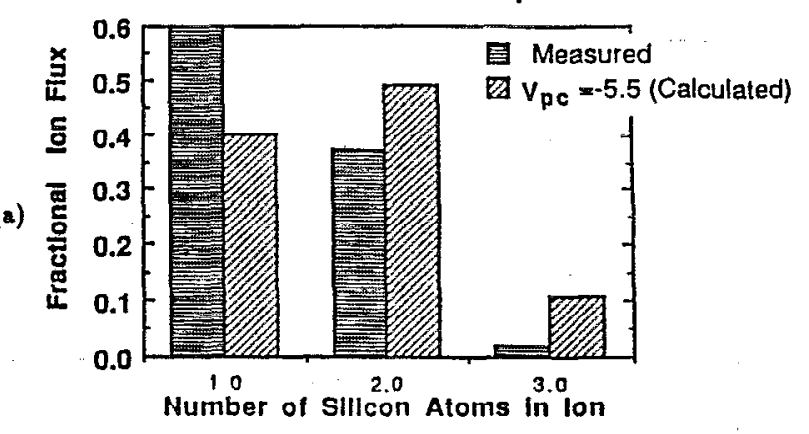

Fractional Ion Flux Comparison

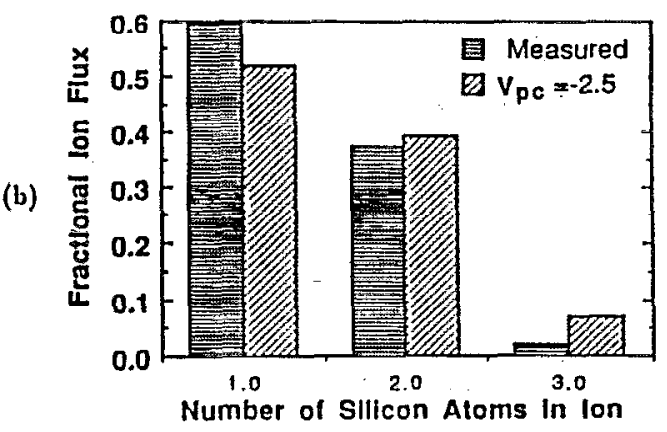

FIG. 8. Effects of reducing the applied potential (and subsequently $\left.\Phi_{\mathrm{pc}}\right)$ by $3 \mathrm{~V}$. (a) Applied $=-580 \mathrm{~V}\left(\Phi_{\mathrm{pc}}=-5.5 \mathrm{~V}\right)$ (experiment). (b) Applied $=-577 \mathrm{~V}\left(\Phi_{\mathrm{pc}}=-2.5 \mathrm{~V}\right)$.

were again calculated. The results of this calculation are shown in Fig. 9 where the fractional ion current varies by up to $15 \%$ of its original value. This shows that the sensitivity of the fractional ion current to changes in chemical rate constants can be appreciable but that the original trend in the $\mathrm{SiH}_{x}^{+}$current remains intact.

Differences between the measured and computed fluxes of the heavier ions can be attributed to the chemical rate

$\mathrm{SiH}_{\mathbf{x}}^{+}$Flux $\mathbf{s} . \mathrm{L}$ and $\mathbf{k}_{1}$

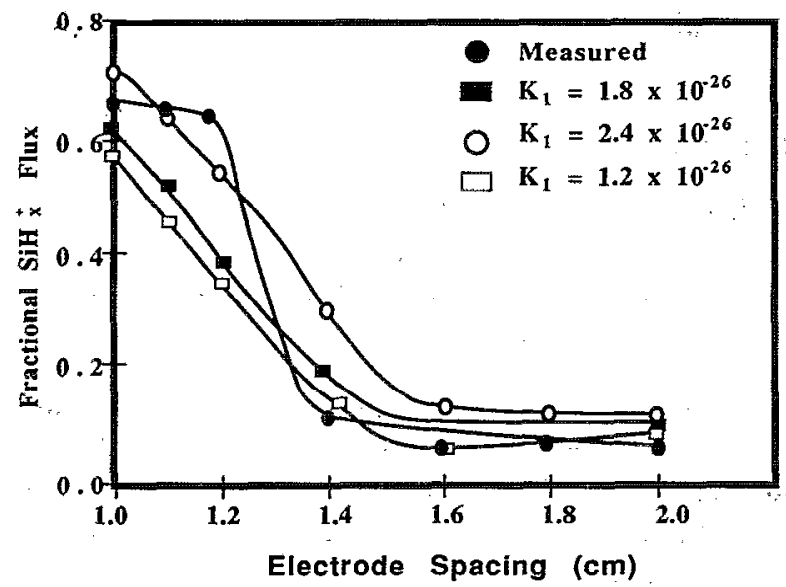

FIG. 9. Calculated fractional ion currents using different values of the rate constant $k_{1}$ (all other rate constants and the clectron temperature remain the same). 


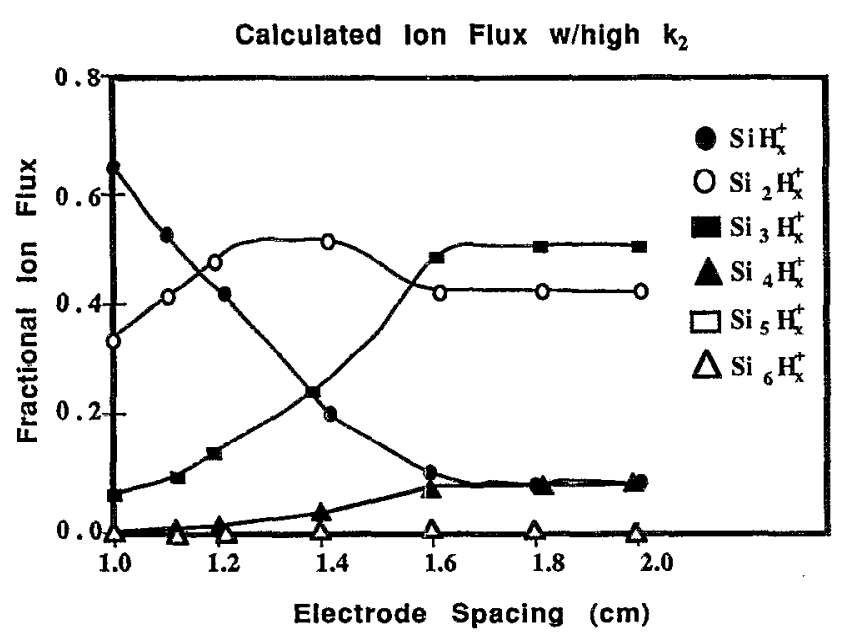

FIG. 10. Effects of increasing the rate constant $k_{2}$ on the fractional ion currents. (a) $k_{2}=5 \times 10^{-11}$ (measured); (b) $k_{2}=1 \times 10^{-10}$.

constants used. Fortunately, for the experimental conditions uscd, therc is a dominant reaction for the formation of heavier ions. This mechanism is

$$
\mathrm{Si}_{2} \mathrm{H}_{7}^{+}+\mathrm{SiH}_{4} \rightarrow \mathrm{Si}_{3} \mathrm{H}_{x}^{+} \text {. }
$$

Effects of changing $k_{2}$ on the ion currents are given in Fig. 10 , where $k_{2}$ is increased by a factor of 2 from the value given in the literature. It can be seen that the trend in the $\mathrm{Si}_{2} \mathrm{H}_{x}^{+}$current now is closer to the measured trends in Fig. 5. This same procedure can be applied to estimate rate constants for the heavier ions.

\section{Proposed model for ion chemistry in the anode sheath}

A possible explanation for the variation in the fractional $\mathrm{SiH}_{x}^{+}$current at the anode will now be presented. For short electrode spacings $(L=1.0 \mathrm{~cm})$, the anode sheath is very close to the negative glow edge. The electrons are still relatively cold since there is only a weak electric field in the negative glow. This yields a relatively low electron temperature and corresponding short anode sheath length and sheath potential drop. For wider electrode spacings $(L>1.1 \mathrm{~cm})$, the electrons are heated by the positive column electric field. As the electron temperaturc increases, so do the anode sheath length (at fixcd electron density) and potential drop. We must now relate the effects of the electron temperature to the number of chemical reactions the ions experience in the anode sheath.

The ratio of the time the ions spend in the anode sheath to the average time for ions to undergo a chemical reaction with silane is

$$
\beta=t_{0} / \tau_{\text {coll }}
$$

where $\tau_{\text {coll }}$ is the mean time for collisions that cause a chemical reaction and $t_{0}$ is the time ions spend in the anode sheath. For this calculation, we will assume all the ions cross the entire anode sheath. The time spent in the anode sheath is given by

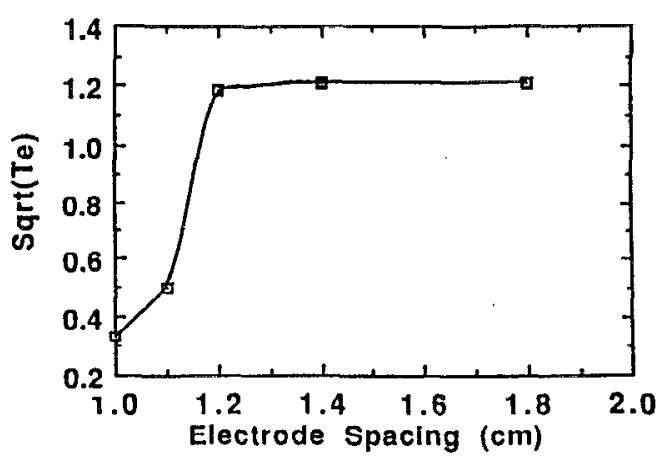

(a)

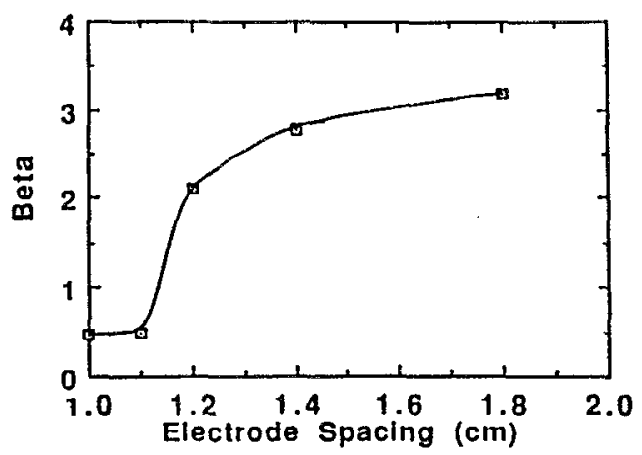

(b)

FIG. 11. (a) The calculated $T_{e}$ as a function of electrode spacing and (b) the ratio of $l_{a f}$ to $\lambda$ of the $\mathrm{SiH}_{x}^{+}$.

$$
t_{0}=\frac{l_{a f}}{\left\langle v_{i}\right\rangle}=\frac{l_{a f}}{\mu_{i}\langle E\rangle},
$$

where $\left\langle v_{i}\right\rangle$ is the average ion velocity, $\mu_{i}$ is the ion mobility and $\langle E\rangle$ is the average electric field. For a linear electric field in the anode sheath this ratio becomes

$$
\beta=\frac{l_{a f^{\prime}}^{2}}{\tau_{\text {coll }} \Phi_{a f}} .
$$

Both $l_{a f}$ and $\Phi_{a f}$ are related to $T_{e}\left(\tau_{\text {coll }}\right.$ is assumed to be constant). In this model, $\Phi_{a f}=3.8 k T_{e} / q$. The relationship between $l_{a f}$ and $T_{e}$ is more complicated. It was assumed that

$$
l_{a f}=5 \sqrt{\epsilon_{0} \kappa T_{e} / q^{2} N_{e}},
$$

where $N_{e}$ is the electron density at the anode sheath edge which is a function of $T_{e}$. Using Eqs. (4), (5), and (6), it may be shown that $N_{e}$ is proportional to $\sqrt{1 / T_{e}}$. Then the anode sheath length is related to the electron temperature by

$$
l_{a f} \propto T_{e}^{3 / 4} .
$$

The number of chemical reactions $\beta$ is given by

$$
\beta \propto l_{a f}^{2} / \Phi_{a f} \propto \sqrt{T_{e}} .
$$

Therefore, at lower temperatures, ions in the anode sheath experience fewer chemical reactions. In Fig. 11(a), the $\sqrt{T_{e}}$ calculated by the code is plotted as a function of electrode spacing. 
We must now find a method to determine the ratio, $\beta$, from the experimental results. To do this, we will examine the decrease in the fractional $\mathrm{SiH}_{x}^{+}$flux. As before, we will assume all the ions travel across the entire anode sheath. If the anode sheath length were negligible, the ions would not experience any chemical reactions and the fractional ion flux at the anode would be 1 . To find the number of chemical reactions experienced by the $\mathrm{SiH}_{x}^{+}$ions from experimental measurements we use the relation

$$
\Gamma=\Gamma_{0} \exp \left(-l_{a f} / \lambda\right),
$$

where $\Gamma$ is the $\mathrm{SiH}_{x}^{+}$flux at the anode, $\Gamma_{0}$ is the $\mathrm{SiH}_{x}^{+}$flux at the anode in the absence of chemical reactions, and $\lambda$ is the mean free path for chemical reactions.

Clearly, the argument of the exponential in (15) is $-\beta$. We can then solve for $\beta$ to get

$$
\beta=-\ln \left(\Gamma / \Gamma_{0}\right) \text {. }
$$

In Fig. 11(b) is a plot of $\beta$ using this derived relation. By comparing Fig. 11(a) with Fig. 11(b), a direct correlation can be observed between the increase in $\sqrt{T_{e}}$ and the increase in $\beta$ from the experiment. This suggests that as $\sqrt{T_{e}}$ increases the number of chemical reactions experienced by the $\mathrm{SiH}_{x}^{+}$increases.

In this derivation, it was implied that all the ions traveled the entire anode sheath. This is not entirely true since the ions are created throughout the anode sheath. The important point is that ions created at the same distance from the anode can have different mean free paths for chemical reactions depending on the anode sheath electric field. This anode sheath electric field is a function of the electrode spacing (via $T_{e}$ at the anode).

\section{CONCLUSION}

In this paper, a model for calculating the motion of the electrons and ions in a dc silane discharge was presented. Good agreement of the fractional ion fluxes at the anode with experimental measurements was found. The effect of increasing the electrode spacing was to increase the electron temperature at the anode. This temperature increase caused the conditions in the anode sheath to change so that the ions would experience more chemical reactions.

A simple analytic model for the behavior of the ion motion in the anode sheath was presented. This model showed trends in the number of chemical reactions experienced by the ions in the anode sheath which were similar to those found in the experiment.

This paper also showed that even if it is not possible to do an electrostatically self-consistent calculation of the particle motion in a discharge, the main aspects of the particle motion can still be modeled. Using these downscaled models, it was possible to describe the principal aspects of a complex silane discharge.

\section{ACKNOWLEDGMENT}

This material is based upon work supported by the National Science Foundation under Grant No. ECD-8721545.

${ }^{1}$ M. J. Kushner, J. Appl. Phys. 54, 4958 (1983); 63, 2532 (1988).

${ }^{2}$ M. J. Druyvesteyn and F. M. Penning, Phys. Rev. 12, 87 (1940).

${ }^{3}$ H. A. Weakleim, R. D. Estes, and P. A. Longeway, J. Vac. Sci. Technol. A 1, 29 (1987).

${ }^{4} \mathrm{H}$. A. Weakleim (private communication).

${ }^{5}$ W. N. G. Hitchon, D. J. Koch, and J. B. Adams, J. Comput. Phys. 83, 79 (1989).

${ }^{6}$ T. J. Sommerer, Ph.D. thesis, University of Wisconsin-Madison, 1990.

${ }^{7}$ Y. Ohmuri, M. Shimozuma, and H. Tagashira, J. Phys. D 19, 1029 (1986).

${ }^{8}$ H. Chatham and A. Gallagher, J. Appl. Phys. 58, 159 (1985).

${ }^{y}$ J. M. S. Henis, G. W. Stewart, and P. P. Gaspar, J. Chem. Phys. 58, 3639 (1973).

${ }^{10}$ J. M. S. Henis, G. W. Stewart, M. K. Tripodi, and P. P. Gaspar, J. Chem. Phys. 57, 389 (1972).

${ }^{11}$ T.-Y. Yu, T. M. H. Cheng, V. Kenpter, and F. W. Lampe, J. Phys. Chem. 76, 3321 (1972).

${ }^{12}$ T. M. H. Cheng, T.-Y. Yu, and F. W. Lampe, J. Phys. Chem. 78, 1184 (1974). 